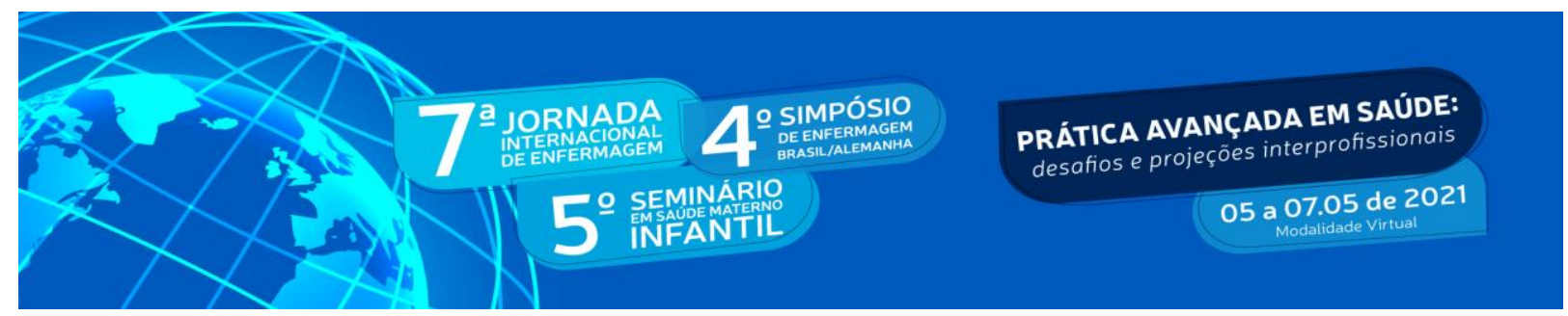

DOI: http://doi.org/10.48195/jie2021-035

\title{
O IMPACTO DA COVID- 19 NA SAÚDE MENTAL DAS PESSOAS: UMA REVISÃO NARRATIVA
}

\author{
Alexandra do Nascimento Damasio Flores ${ }^{1}$; Larissa Dias Antunes²; Daiana Foggiato de \\ Siqueira ${ }^{3}$
}

\section{RESUMO}

O objetivo do estudo é identificar as publicações científicas da enfermagem acerca do impacto da Covid19 na saúde mental das pessoas. Trata-se de uma revisão narrativa da literatura. O levantamento dos artigos científicos utilizados foi realizado a partir de pesquisa eletrônica na base de dados Literatura Latino-Americana e do Caribe em Ciências da Saúde, utilizando os descritores para o levantamento dos artigos: "enfermagem" AND "saúde mental" AND "pandemias" por meio do campo "Descritor de assunto" foram encontradas 24 produções na LILACS, destas seis compuseram o estudo. Observou-se um aumento significativo de pessoas desenvolvendo problemas relacionados à saúde mental durante a pandemia, entre elas, os profissionais que atuam na linha de frente. Com isso, é fundamental que haja maiores pesquisas a respeito da saúde mental neste período, para que a partir destas, evite-se o adoecimento psíquico da população, e também dos profissionais de saúde.

Palavras Chave: Enfermagem; Isolamento Social; Pandemias.

\begin{abstract}
The aim of the study is to identify scientific nursing publications about the impact of Covid-19 on people's mental health. This is a narrative review of the literature. The survey of the scientific articles used was carried out through electronic research in the Latin American and Caribbean Literature in Health Sciences database, using the descriptors for the survey of the articles: "nursing" AND "mental health" AND "pandemics" through the "Subject descriptor" field, 24 productions were found at LILACS, of these six made up the study. There was a significant increase in people developing problems related to mental health during the pandemic, including professionals working on the front line. Thus, it is essential that there is more research on mental health in this period, so that from these, the population's psychological illness, and also of health professionals, is avoided.
\end{abstract}

Keywords: Nursing; Social Isolation; Pandemics.

\section{INTRODUÇÃO}

\footnotetext{
${ }^{1}$ Acadêmica do Curso de Enfermagem - UFSM. alexandrad2102@gmail.com

${ }^{2}$ Acadêmica do Curso de Enfermagem - UFSM. Bolsista PIBIC. larissadiasanttunes@ gmail.com

${ }^{3}$ Orientadora Dra. Profa. do Departamento de enfermagem - UFSM. daianasiqueira@ yahoo.com.br
} 


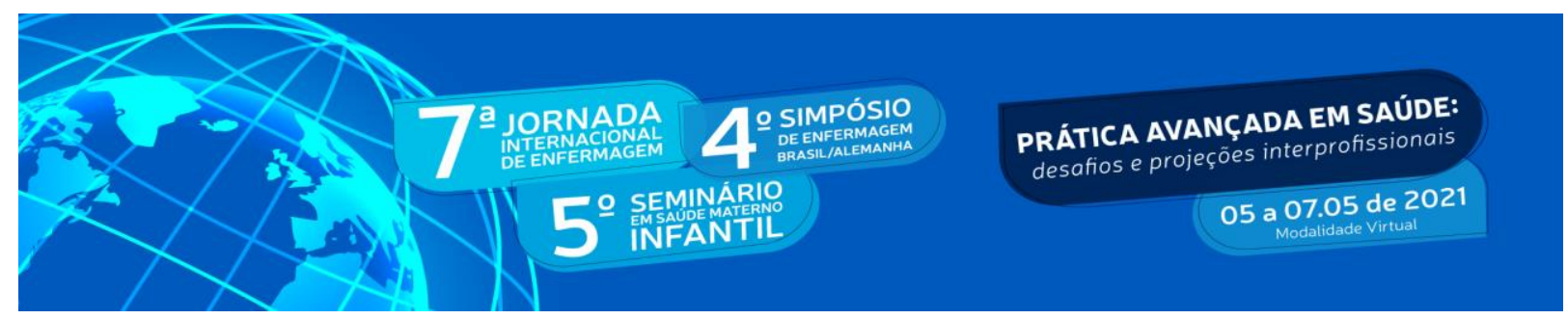

A doença denominada como COVID- 19, surge do Novo Coronavírus, sendo o responsável o SARS- CoV-2, o vírus é o causador de inúmeros casos de pneumonia e insuficiência respiratória. Tem como característica a alta e rápida taxa de transmissibilidade, assim como o seu nível de letalidade é considerado variável, havendo a necessidade de medidas e ações que diminuam o contágio no cenário de transmissão comunitária, visando maiores estudos quanto ao vírus e suas consequências, sendo necessário o isolamento social da população e evitar as aglomerações, devido a sua alta velocidade de propagação (BRASIL, 2020).

Sabe- se que a equipe de enfermagem tem como envolvimento um trabalho na linha de frente ao enfrentamento da Covid-19. Dessa forma, tem-se maior potencial de desenvolver impactos negativos nas questões psicossociais e psicossomáticos, proporcionando manifestações de ansiedade e depressão nos profissionais em ambiente ocupacional (DAL'BOSCO et al.; 2020).

Destaca- se, a necessidade de difundir estudos nessa temática dos impactos que a Covid19 esta causando na população voltados a área da saúde mental, a fim de atingir e conscientizar cada vez mais profissionais da saúde e consequentemente usuários. Dessa forma, se faz possível e necessário desenvolver práticas preventivas voltadas à promoção da saúde mental.

\section{OBJETIVO}

O objetivo do presente estudo é identificar as publicações científicas da enfermagem acerca do impacto da Covid-19 na saúde mental das pessoas.

\section{METODOLOGIA}

Trata-se de uma revisão narrativa da literatura, onde os artigos de revisão, assim como outras categorias de artigos científicos, são uma forma de pesquisa que utilizam de fontes de informações bibliográficas ou eletrônicas para obtenção de resultados de pesquisas de outros autores, com o objetivo de fundamentar teoricamente um determinado objetivo (ROTHER, 2007). 


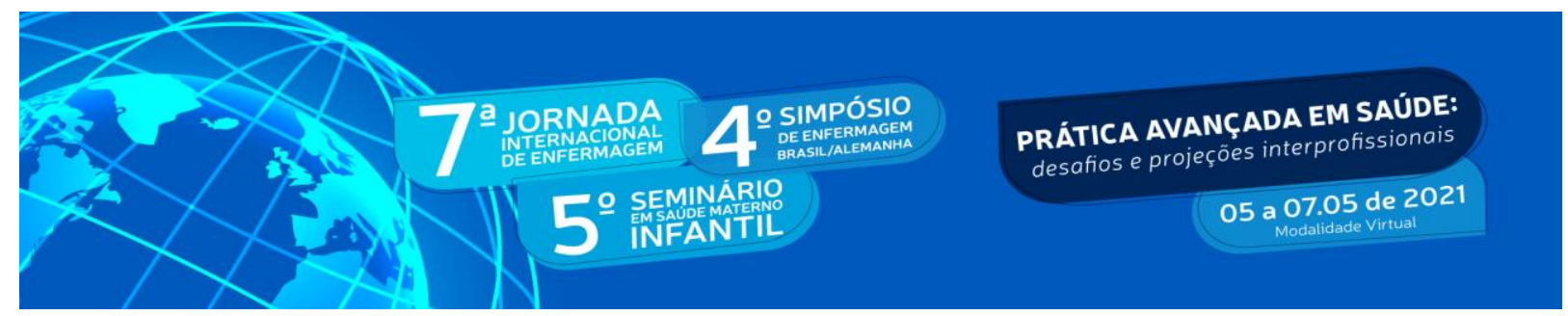

O presente trabalho tem finalidade de sintetizar e resumir uma gama de publicações científicas. A revisão foi construída a partir da questão norteadora: Qual a produção cientifica na enfermagem sobre o impacto da Covid-19 na saúde mental das pessoas?

O levantamento de artigos científicos foi realizado a partir de pesquisa eletrônica na base de dados Literatura Latino-Americana e do Caribe em Ciências da Saúde (LILACS). O acesso ocorreu no mês de março de 2020. Os descritores utilizados para o levantamento dos artigos, de acordo com os Descritores em Ciências da Saúde (DeCS), foram: "enfermagem” AND "saúde mental" AND "pandemias" por meio do campo "Descritor de assunto" foram encontradas 24 produções na LILACS.

Para a inclusão das produções determinou-se que fossem artigos científicos, disponíveis completos on-line, gratuitos e nos idiomas inglês, português e espanhol. Foram excluídas as publicações que não respondessem a pergunta da revisão, artigos de reflexão, relatos de experiência e revisões bibliográficas. As produções repetidas nas bases de dados foram computadas uma única vez. Desta maneira, foram selecionados 6 artigos na LILACS.

Figura 01: Fluxograma da seleção dos artigos baseado nas etapas de identificação, triagem, elegibilidade e inclusão. Santa Maria, Rio Grande do Sul, Brasil, 2021. 

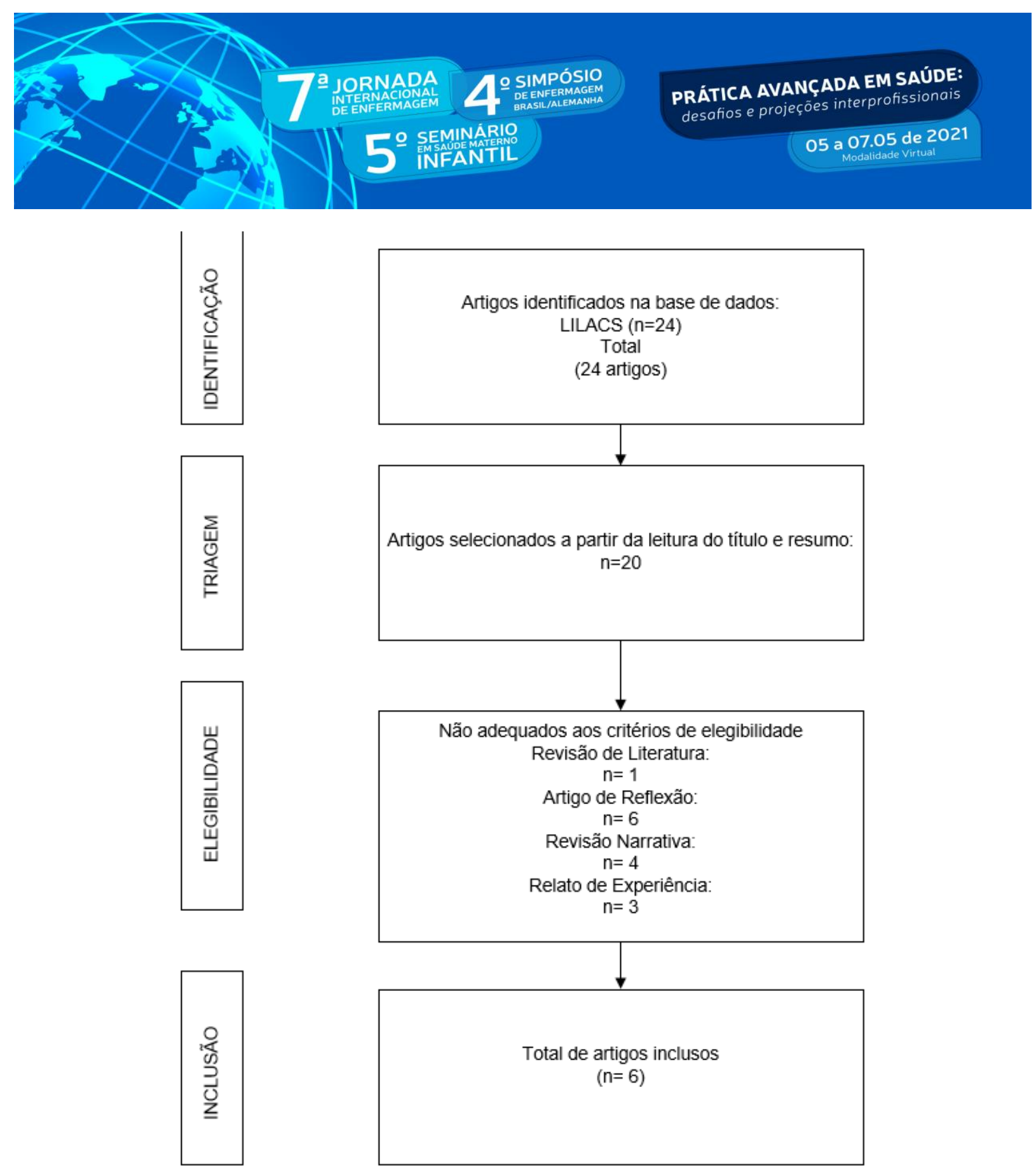

Fonte: Dados coletados pelo autor.

\section{RESULTADOS E DISCUSSÃO}

O novo coronavírus (Covid- 19) tem como característica a alta velocidade de propagação, como resultado rapidamente o vírus se propagou mundialmente, tornando- se uma pandemia. Exigiu que o Ministério da Saúde (MS) priorize e enfatiza medidas de prevenção, 


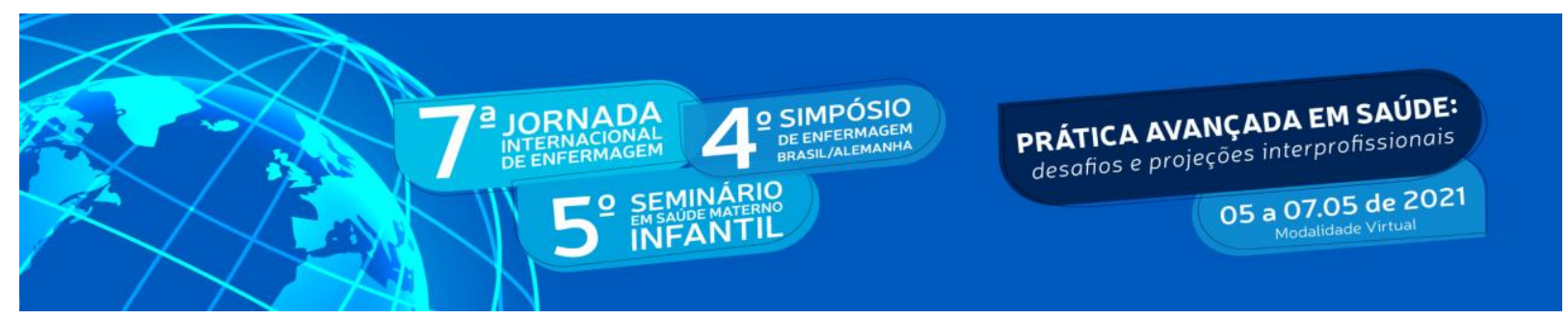

contenção e controle do vírus, com intuito de evitar o colapso na saúde e impedir milhões de mortes (SANTOS; RODRIGUES, 2020).

Notou-se um aumento significativo de pessoas que desenvolveram depressão, ansiedade, pânico, decorrente do período no qual esta se vivendo, proporcionando ao desenvolvimento de um comportamento focado na autoproteção (FERNANDES; ARAÚJO, 2020).

O isolamento social, necessário nesta pandemia, favorece a predominância do adoecimento psíquico na sociedade, tendo o surgimento e aumento de sentimentos negativos como, solidão, raiva, frustração, medo da morte, tristeza e irritabilidade. Com a quarentena pode-se observar a quebra de rotina das pessoas, mostrando que a interação social e emocional é essencial para a prevenção e conservação da saúde mental (SANTOS; RODRIGUES, 2020).

Dentre a população, têm-se os profissionais da saúde atuantes na linha de frente do enfrentamento ao Covid-19. O colapso na saúde, carga horária extensa e sobrecarregada, descaso com a falta de descanso, propicia maior estresse e irritabilidade, refletindo durante a sua jornada de trabalho, realizando um atendimento desanimado, com ansiedade e podendo desenvolver depressão e nos casos mais críticos Síndrome de Burnout (DAL’BOSCO et al.; 2020).

Assim como profissionais da saúde, pessoas com Transtornos Mentais (TM) e com comorbidades devem ser observadas com maior atenção. A quarentena e o isolamento social favorecem aos pacientes com adoecimento psíquico terem um surto na saúde mental, propiciando para que caso obtenha a doença, a adesão ao tratamento tenha maior dificuldade (CASTRO-DE-ARAUJO; MACHADO, 2020).

É notório o impacto na saúde mental da população devido ao reflexo dos acontecimentos proporcionados pela pandemia, enfatizando para a importância da segurança psicológica tanto dos pacientes em situação de risco quanto dos profissionais da saúde atuantes na linha de frente. Indicadores pré-estabelecidos como necessidade constante de realizar ligações para familiares, atitudes de isolamento, exaustão física e busca frequente por informações sobre a doença, são alguns dos exemplos que deve- se atentar, para amenizar o impacto psicológico é necessário monitorar e caso necessário encontrar ações específicas (ALVAREZ; ALMAGUER; SANTOS, 2020). 


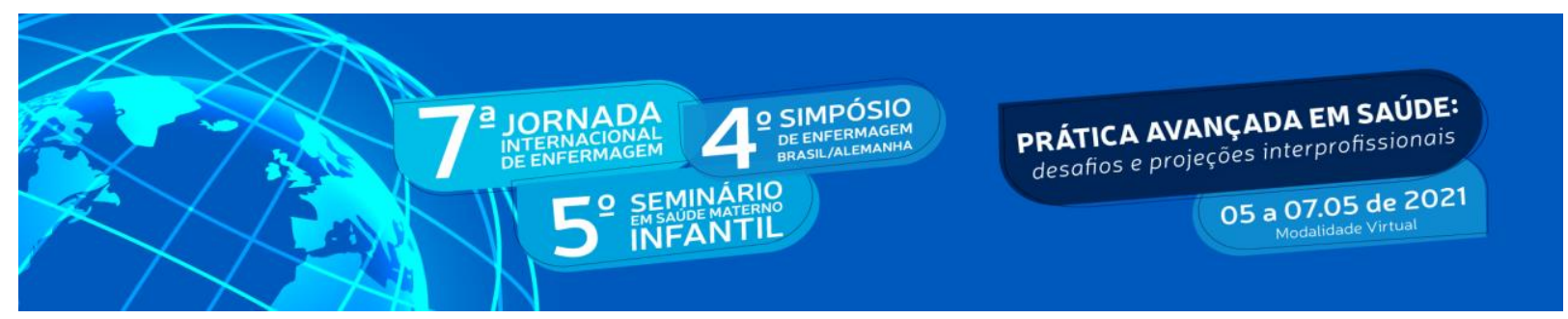

Para a manutenção da saúde mental da população, durante este período no qual esta se vivendo, é essencial desenvolver ações e protocolos não só voltados a contenção do contágio do vírus, assim como, também a promoção da saúde mental, favorecendo ao acolhimento humanizado (SANTOS; RODRIGUES, 2020).

A pandemia gerada pelo novo coronavírus, necessitou de as pessoas readequarem as suas rotinas devido ao isolamento social necessário, logo, proporcionou grandes reflexões e novas percepções quanto ao essencial das suas vidas. Gradativamente os impactos gerados pela doença estão aparecendo, sendo primordial atentar- se aos campos não só físicos como também no âmbito da saúde mental, sendo fundamental uma assistência humanizada a toda população.

\section{CONCLUSÃO}

Os impactos referentes à saúde mental da população estão gradativamente aumentando com o passar do tempo, sendo por motivo de medo, referente a doença Covid- 19, sendo pelo medo instaurado a morte ou pela necessidade de realizar o isolamento social.

Atentar-se a determinados grupos como profissionais da saúde, priorizando a sua saúde mental, proporciona um profissional menos estressado, com menores chances de desenvolver síndrome psíquica, favorecendo um melhor atendimento para enfrentar na linha de frente da doença.

É fundamental que ocorra maiores pesquisas a respeito da saúde mental neste período, desenvolvendo protocolos, ações e medidas que auxiliem na promoção da saúde, evitando o maior desenvolvimento de pessoas em adoecimento psíquico.

\section{REFERÊNCIAS}

ALVAREZ, A. K. G.; ALMAGUER, A. Y. C.; SANTOS, E. D. Z. Gestión de seguridad psicológica del personal sanitario en situaciones de emergencia por COVID-19 en el contexto hospitalario o de aislamiento. Revista Cubana de Enfermería, v. 36, n. 2, p. 1-19, 2020.

BRASIL. Ministério da Saúde. Secretaria de Atenção à Saúde. Departamento de Ações Programáticas Estratégicas. Caderno HumanizaSUS. Brasília, 2015.

BRASIL. Protocolo de manejo clínico do coronavírus (Covid-19) na Atenção Primária à

Saúde. Brasília: Ministério da Saúde; 2020. Disponível em: 


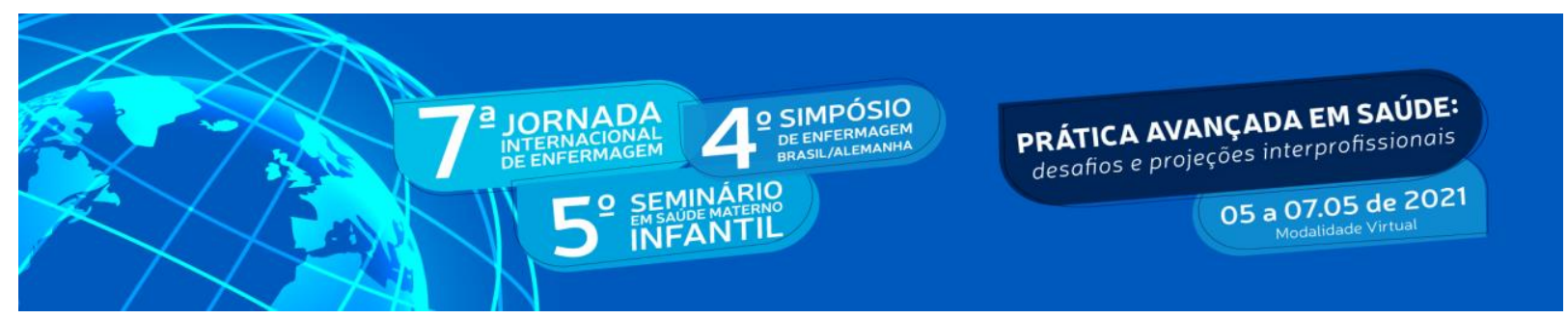

https://saude.rs.gov.br/upload/arquivos/202004/14140606-4-ms-protocolomanejo-apsver07abril.pdf

CASTRO-DE-ARAUJO, L. F. S.; MACHADO, D. B. Impact of COVID-19 on mental health in a Low and Middle-Income Country. Revista Ciência \& Saúde Coletiva, Manguinhos, v. 25, p. 2457-2460, 2020.

DA CRUZ SANTOS, G. et al. Covid-19 nas prisões: efeitos da pandemia sobre a saúde mental de mulheres privadas de liberdade. Revista Baiana de Enfermagem, Salvador, v. 34, 2020.

DAL'BOSCO, E. B. et al. A saúde mental da enfermagem no enfrentamento da COVID-19 em um hospital universitário regional. Revista Brasileira de Enfermagem, Brasília, v. 73, 2020.

FERNANDES, M. A.; ARAÚJO, A. A. C. Empatía y salud mental en el contexto de la pandemia por COVID-19. Revista Cubana de Enfermería, v. 36, n. 2, p. 1-3, 2020.

ROTHER, E.T; Revisão sistemática X revisão narrativa. Revista Acta Paulista de Enfermagem. São Paulo, v.20 n.2, São Paulo Apr./June 2007.

SANTOS, M. F.; RODRIGUES, J. F. S. COVID-19 e repercussões psicológicas durante a quarentena e o isolamento social: uma revisão integrativa. Revista Nursing Brasil, São Paulo, p. 4095-4100, 2020. 